\title{
Formation, Phase Composition and Memristive Properties of Titanium Oxide Nanodots ${ }^{\dagger}$
}

\author{
Vadim I. Avilov *, Roman V. Tominov, Denis A. Suharevich, Ivan S. Ugrumov, Maksim S. Solodovnik, \\ Vladimir A. Smirnov and Oleg A. Ageev
}

Citation: Avilov, V.I.; Tominov, R.V.; Suharevich, D.A.; Ugrumov, I.S.; Solodovnik, M.S.; Smirnov, V.A.; Ageev, O.A. Formation, Phase Composition and Memristive Properties of Titanium Oxide Nanodots. Mater. Proc. 2021, 4, 44 https://doi.org/10.3390/ IOCN2020-07848

Academic Editors: Ana María Díez-Pascual, Antonio Di Bartolomeo and Guanying Chen

Published: 15 November 2020

Publisher's Note: MDPI stays neutral with regard to jurisdictional claims in published maps and institutional affiliations.

Copyright: $@ 2021$ by the authors. Licensee MDPI, Basel, Switzerland. This article is an open access article distributed under the terms and conditions of the Creative Commons Attribution (CC BY) license (http://creativecommons.org/licenses/by/4.0/).
Institute of Nanotechnologies, Electronics and Electronic Equipment Engineering, Southern Federal University, 347922 Taganrog, Russia; tominov@sfedu.ru (R.V.T.); suharevich@sfedu.ru (D.A.S.); iugryumov@sfedu.ru (I.S.U.), solodovnikms@sfedu.ru (M.S.S.); vasmirnov@sfedu.ru (V.A.S.); ageev@sfedu.ru (O.A.A.)

* Correspondence: avilovvi@sfedu.ru; Tel.: +7-863-437-1629

+ Presented at the 2nd International Online-Conference on Nanomaterials, 15-30 November 2020; Available online: https://iocn2020.sciforum.net/.

\begin{abstract}
In this work, the titanium oxide nanodots arrays formation was carried out. A currentvoltage characteristics study of nanodots showed that the resulting structure switches between high resistance and low resistance states. Additionally, we performed a numerical simulation of the formation of oxide nanodots obtained by the local anodic oxidation method, the results of which showed that the $\mathrm{TiO}_{2}$ phase dominates on the formed oxide surface. As the oxide goes deeper into the bulk, the $\mathrm{Ti}_{2} \mathrm{O}_{3}$ and $\mathrm{TiO}$ phases appear and the $\mathrm{TiO}$ phase prevails near the metal/oxide interface. To confirm the simulation results, the titanium oxide nanodots phase composition was studied by the XPS method, which confirmed the theoretical results.
\end{abstract}

Keywords: nanotechnology; scanning probe microscopy; local anodic oxidation; titanium oxide nanostructures; nanodots; effect of resistive switching

\section{Introduction}

The increase in the modern electronics productivity is limited by the CMOS structure minimum size [1-3]. Furthermore, miniaturization is impossible due to an increased leakage power consumption, reduced reliability and a more complex fabrication process. Therefore, increasingly attention is paid to the development and research of new nanoelectronic elements with small characterizing dimensions, as well as high performance and speed. The recently discovered resistive switching effect has led to the development of memristor switching between high (HRS) and low (LRS) resistance states [4,5]. Such structures can be used both in memory elements and in logical computation operations. In addition, there is an idea of computation-in-memory architecture that combines the functions of computing and data storage in one integrated circuit [6].

An analysis of the literature has shown that titanium oxide nanostructures are most preferable when creating memristors with good characteristics in terms of speed and energy efficiency, while the best characteristics are achieved when a nonuniform stoichiometric composition of titanium oxide is formed [7,8]. The method of local anodic oxidation is the most promising for the creation of memristors based on titanium oxide since the resulting structures exhibit a forming-free memristor effect and can be used to create memory elements or perform logical operations $[9,10]$.

Therefore, the actual task and goal of this work is the experimental and theoretical study of the formation and resistive switching dependencies of titanium oxide nanodots obtained by the method of local anodic oxidation (LAO). 


\section{Methods}

For scientific research, we used a $20 \mathrm{~nm}$ thick titanium film obtained by magnetron sputtering on the surface of a $\mathrm{Si} / \mathrm{SiO}_{2}$ substrate. Using a scanning probe microscope Solver P47 Pro (NT-MDT, Russia), oxide nanodots (OND) were formed by the LAO method by applying voltage pulses between the probe and the titanium film.

A numerical model of the formation of OND was also developed, which made it possible to calculate the nanodot profile at the given local anodic oxidation parameters. In addition, this model makes it possible to calculate the distribution of titanium oxide phases in the obtained nanodot volume.

To confirm the obtained oxide phases distribution theoretical results, an XPS analysis of a titanium oxide nanodots array with depth etching was carried out by using a combined spectrometer for quantitative imaging and surface analysis of ESCALAB 250 (Thermo Scientific, Waltham, MA, USA) with monochromatizating of X-ray AlK $\alpha$-line.

The current-voltage characteristics were studied in the mode of current spectroscopy of a scanning probe microscope in the contact mode.

\section{Results and Discussion}

In the LAO technological parameters influence studies on the OND geometric parameters, titanium oxide nanodots arrays were formed, as well as numerical simulation of geometric dimensions (Figure 1). It was shown that the theoretical results are in good agreement with the experimental study results; an increase in the voltage pulse amplitude and air humidity in the technological chamber leads to an increase in the OND height and diameter. This is explained by the increase in the ions number formed due to the decomposition of water molecules in the gap between the probe and the OND surface.

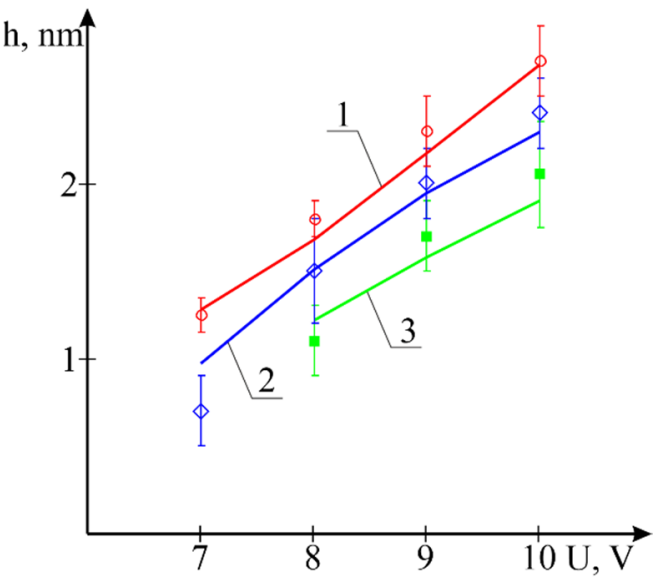

(a)

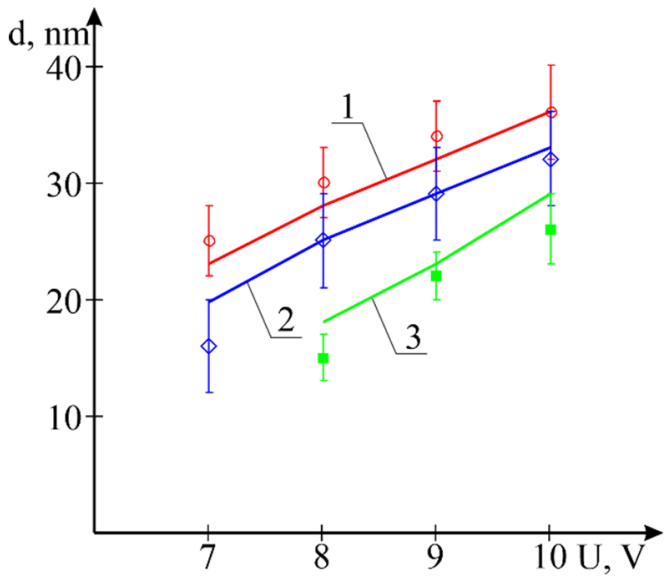

(b)

Figure 1. Experimental (points) and theoretical (lines) OND height (a) and diameter (b) dependence on the applied voltage pulse amplitude at a relative humidity level of $1-90 \%, 2-70 \%$ and $3-50 \%$.

In addition, the obtained model makes it possible to calculate the titanium oxide phases distribution in the formed oxide volume (Figure 2). It was shown that the $\mathrm{TiO}$, $\mathrm{Ti}_{2} \mathrm{O}_{3}$ and $\mathrm{TiO}_{2}$ phases can be formed in the $\mathrm{LAO}$ process. Moreover, near the nanodot surface, the $\mathrm{TiO}_{2}$ phase predominates; as one moves deeper into the oxide, the fraction of the $\mathrm{TiO}_{2}$ phase decreases and the fraction of the $\mathrm{Ti}_{2} \mathrm{O}_{3}$ and $\mathrm{TiO}$ phases increases. The $\mathrm{TiO}$ phase predominates near the oxidized metal surface. These dependences are explained by the fact that during LAO oxidizer ions move in an external electric field through the oxide volume to the metal surface. In this case, the oxygen concentration is highest near the oxide surface, the $\mathrm{TiO}_{2}$ phase is formed and near the surface of the metal, the oxygen ions concentration is the lowest and the $\mathrm{TiO}$ phase is formed. 

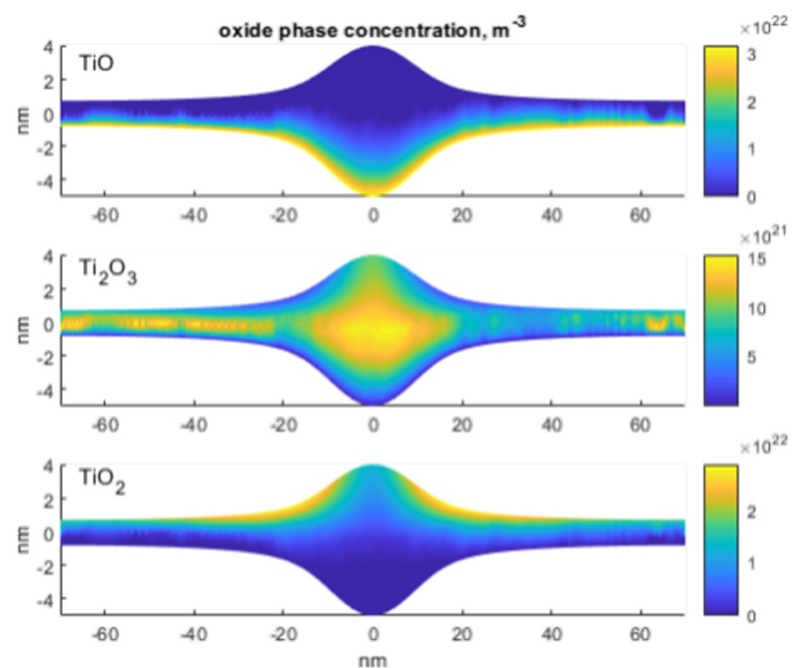

Figure 2. Titanium oxide phases distribution in the nanodot volume by numerical model.

To confirm the obtained experimental results, a titanium oxide nanodots array XPS was carried out (Figure 3), which showed good correlation with the obtained theoretical results.

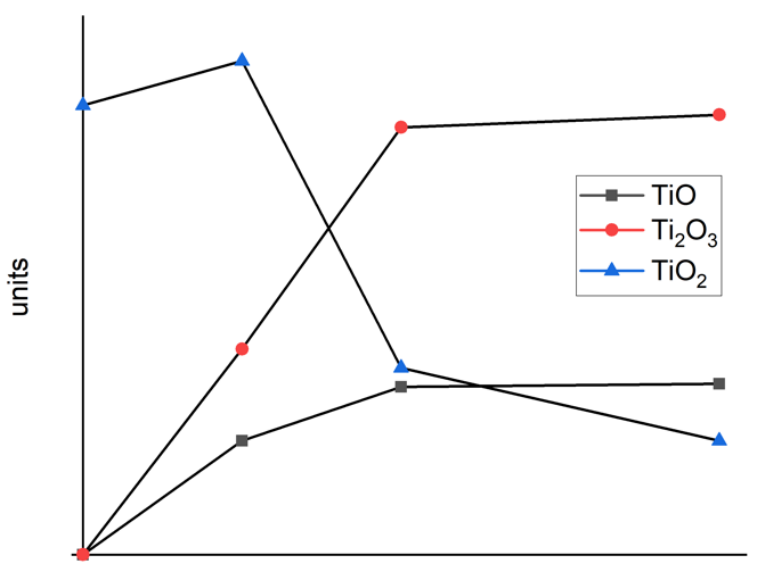

etch time, $\mathrm{s}$

Figure 3. Titanium oxide phases distribution in the nanodot volume by XPS.

The current-voltage characteristics study of the OND showed that they exhibit a

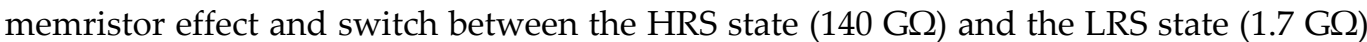
(Figure 4) without additional forming operation. 


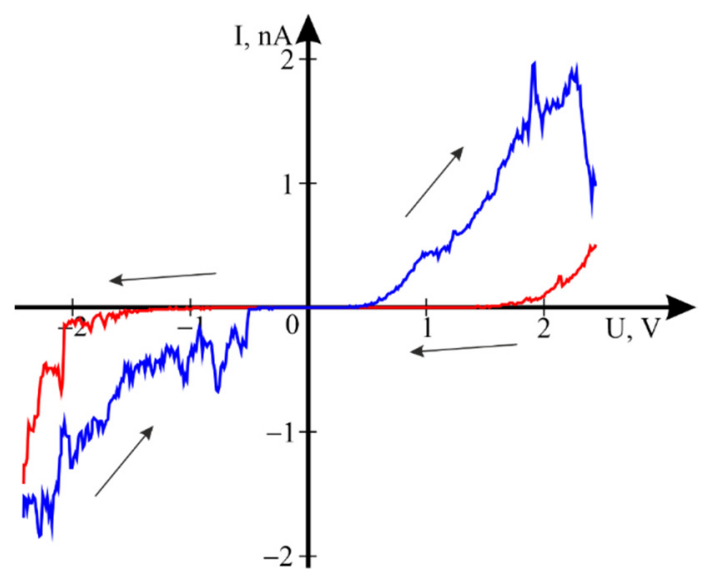

Figure 4. Titanium oxide nanodots current-voltage characteristics.

\section{Conclusions}

Thus, in this work, titanium oxide nanodots formation theoretical and experimental studies by the local anodic oxidation method and phase composition were carried out. The electrical characteristics study showed that the resulting structures exhibit a formingfree memristor effect. The results can be used in the development of technological processes for the formation of elements of nanoelectronics, as well as elements of resistive memory based on oxide nanoscale structures.

Funding: This work was supported by RFBR according to the research project No. 19-29-03041 mk and by grant of the President of the Russian Federation No. MK-767.2020.8.

Institutional Review Board Statement: Not applicable.

Informed Consent Statement: Not applicable.

Data Availability Statement: Not applicable.

\section{References}

1. Jaiswal, A.; Chakraborty, I.; Agrawal, A.; Roy, K. 8T SRAM cell as a multibit dot-product engine for beyond von neumann computing. IEEE Trans. Very Large Scale Integr. (VLSI) Syst. 2019, 27, 2556-2567.

2. Smith, L.S. Neuromorphic systems: Past, present and future. Brain Inspired Cogn. Syst. 2008, 1, 167-182.

3. Jo, S.H.; Chang, T.; Ebong, I.; Bhadviya, B.B.; Mazumder, P.; Lu, W. Nanoscale memristor device as synapse in neuromorphic systems. Nano Lett. 2010, 10, 1297-1301.

4. Prezioso, M.; Merrikh-Bayat, F.; Hoskins, B.D.; Adam, G.C.; Likharev, K.K.; Strukov, D.B. Training and operation of an integrated neuromorphic network based on metal-oxide memristors. Nature 2015, 521, 61.

5. Avilov, V.I.; Smirnov, V.A.; Fedotov, A.A.; Tominov, R.V.; Sharapov, N.A.; Polupanov, N.A. Formation of memristor nanostructures for RRAM memory by local anodic oxidation. IOP Conf. Ser. Mater. Sci. Eng. 2018, 443, 012004.

6. Xie, L.; Du Nguyen, H.A.; Taouil, M.; Hamdioui, S.; Bertels, K. A Mapping Methodology of Boolean Logic Circuits on Memristor Crossbar. Aided Des. Integr. Circuits Syst. 2018, 37, 311-323.

7. Bousoulas, P.; Giannopoulos, J.; Giannakopoulos, K.; Dimitrakis, P.; Tsoukalas, D. Memory programming of $\mathrm{TiO}_{2-x}$ films by Conductive Atomic Force Microscopy evidencing filamentary resistive switching. Appl. Surf. Sci. 2015, 32, 55-61.

8. Lu, W.; Wong, L.M.; Wang, S.; Zeng, K. Probing electrochemically induced resistive switching of $\mathrm{TiO}_{2}$ using $\mathrm{SPM}_{\text {techniques. }}$ Phys. Chem. 2017, 19, 31399-31409.

9. Avilov, V.I.; Kolomiytsev, A.S.; Tominov, R.V.; Alyabyeva, N.I.; Bykova, E.M. Investigation of the electrode material influence on the titanium oxide nanosize structures memristor effect. IOP Conf. Ser. J. Phys. Conf. Ser. 2018, 1124, 022019.

10. Avilov, V.I.; Tominov, R.V.; Sharapov, N.A.; Smirnov, V.A.; Ageev, O.A. Local Anodic Oxidation Proceses Influence and Temterature Stability on the Memristive Propherties of Titanium Oxide Nanostructures for ReRAM Development Moscow Workshop on Electronic and Networking Technologies. In Proceedings of the 2020 Moscow Workshop on Electronic and Networking Technologies (MWENT), Moscow, Russia, 11-13 March 2020; p. 9067405. 